\title{
ChemComm
}

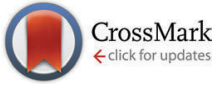

Cite this: Chem. Commun., 2017, 53, 905

Received 16th September 2016, Accepted 14th December 2016

DOI: $10.1039 / c 6 c c 07553 c$

www.rsc.org/chemcomm

\section{Asymmetric rhenium tricarbonyl complexes show superior luminescence properties in live cell imaging $\dagger$}

\author{
Lukasz J. Raszeja, $\ddagger^{a}$ Daniel Siegmund, $\ddagger^{a}$ Anna L. Cordes, ${ }^{a}$ Jörn Güldenhaupt, \\ Klaus Gerwert, ${ }^{\mathrm{b}}$ Stephan Hahn ${ }^{\mathrm{c}}$ and Nils Metzler-Nolte ${ }^{\star a}$
}

\begin{abstract}
The synthesis and photophysical properties of a novel series of rhenium tricarbonyl complexes based on tridentate phenanthridinylcontaining ligands are described. Photophysical data reveal beneficial luminescence behaviour especially for compounds with an asymmetric ligand set. These advantageous properties are not limited to organic solvents, but indeed also improved in aqueous solutions. The suitability of our new rhenium complexes as potent imaging agents has been confirmed by fluorescence microscopy on living cancer cells, which also confirms superior long-time stability under fluorescence microscopy conditions. Colocalisation studies with commercial organelle stains reveal an accumulation of the complexes in the endoplasmic reticulum for all tested cell lines.
\end{abstract}

A basic idea of medicinal bioinorganic chemistry is to get a deeper understanding of fundamental cellular processes. This includes also the quest for new insights into the modes of action of metal based drugs and the identification of their subcellular targets. For this purpose, fluorescence microscopy is an indispensable tool in order to verify the cellular distribution or accumulation of biologically active compounds. Although, it is common to use organic dyes for fluorescence microscopy investigations and biological assays, emissive metal complexes have gained an increasing interest in this field. ${ }^{1-7}$ Especially $\mathrm{d}^{6}$ low spin complexes of Ir(III), Ru(II) and Re(I) show highly promising photophysical properties. In general, these complexes benefit from large Stokes shifts, long emission life times and enhanced photostability. ${ }^{8,9}$

\footnotetext{
${ }^{a}$ Faculty of Chemistry and Biochemistry, Inorganic Chemistry I - Bioinorganic Chemistry, Ruhr-University Bochum, Universitätsstr. 150, 44801 Bochum, Germany. E-mail: Nils.Metzler-Nolte@rub.de

${ }^{b}$ Faculty of Biology and Biotechnology, Chair of Biophysics, Ruhr-University Bochum, Universitätsstr. 150, 44801 Bochum, Germany

${ }^{c}$ Ruhr-University Bochum, Zentrum für Klinische Forschung (ZKF),

Universitätsstraße 150, 44780 Bochum, Germany

$\dagger$ Electronic supplementary information (ESI) available: Expt. details; synthesis and characterizing data of the new compounds; crystallographic data of 4, 5 and 6 (CCDC 853881-853883); cell culture and fluorescence microscopy experiments. For ESI and crystallographic data in CIF or other electronic format see DOI: $10.1039 / \mathrm{c} 6 \mathrm{cc} 07553 \mathrm{c}$

\$ These authors contributed equally to this work.
}

Furthermore, their photophysical behaviour can be tuned by the modification and variation of their ligands. Published emissive $\operatorname{Re}(\mathrm{I})$ complexes are frequently based on the $f a c-\operatorname{Re}(\mathrm{CO})_{3}$ core where bipyridine or phenanthroline derivatives ${ }^{10-19}$ or tridentate chelating ligands ${ }^{20-27}$ occupy the remaining coordination sites.

Herein we report on the favourable photophysical properties of novel rhenium tricarbonyl complexes, which are based on phenanthridinyl(phen)-containing tridentate ligands. Most importantly, these complexes exhibit their beneficial luminescence behaviour not only in degassed acetonitrile but also in air-equilibrated water. Further, these advantageous luminescence properties are underlined by the successful application of the described complexes as imaging agents in live cell fluorescence microscopy.

The ligands designed for our studies unite two important characteristics for potential imaging applications. Their phenbased extended $\pi$-system ensures the availability of electronically accessible ligand orbitals for MLCT or IL transitions which are crucial for the luminescence behaviour of the resulting complexes. Secondly, being benzoic acid derivatives, ester hydrolysis and subsequent conjugation to a variety of biologically active molecules or drugs enables the usage of these complexes as luminescent tags for tracing such bioactive compounds. ${ }^{28}$ To tune and evaluate the influence of phen on the photophysical properties, one of the phen moieties in the symmetrical ligand 1 is replaced by a well-known picolyl or quinolyl moiety respectively, leading to unsymmetrical ligands $\mathbf{2}$ and $\mathbf{3}$. Symmetric ligand 1 was prepared in one step by the reaction of 6-(chloromethyl)phenanthridine with 4-(aminomethyl)benzoic acid methyl ester. ${ }^{28}$ The asymmetric ligands 2 and 3 were synthesized through the sequential reaction of 4-(aminomethyl)benzoic acid methyl ester with 2-picolyl chloride or 2-(chloromethyl)quinoline followed by the reaction with 6-(chloromethyl)phenanthridine. Finally, the ligands were reacted with the $\left[\mathrm{Re}(\mathrm{CO})_{3}\left(\mathrm{H}_{2} \mathrm{O}\right)_{3}\right] \mathrm{Br}$ precursor under microwave conditions to yield the desired rhenium tricarbonyl complexes 4-6 (Fig. 1). Synthetic details and full characterization of the compounds, including single crystal X-ray structures for 4-6 can be found in the ESI. $\dagger$ 
<smiles>COC(=O)c1ccc(CN(Cc2nn(Cc3ccccc3)c3ccccc23)c2cccc3ccccc23)cc1</smiles><smiles>COC(=O)c1ccc(CN(Cn2cccn2)c2ccccc2-c2ccccc2)cc1</smiles><smiles>COC(=O)c1ccc(CN(Cc2ccc3ccccc3n2)c2cccc3ccccc23)cc1</smiles>
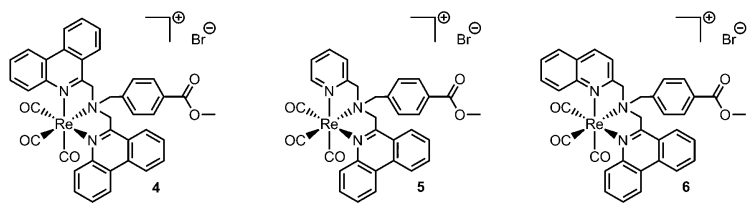

Fig. 1 Novel tridentate ligands and their corresponding rhenium tricarbonyl complexes.

All rhenium complexes show a characteristic broad UV/Vis absorption in the range between $300 \mathrm{~nm}$ and $380 \mathrm{~nm}$. These absorption bands are predominantly defined by the phenanthridinyl moiety and are assigned to intraligand (IL) $\pi \rightarrow \pi^{*}$ transitions (Fig. 2). ${ }^{29-31}$ The energy of those ligand-centered absorption bands do not vary considerably compared to pure ligands, but a substantial 3-4 fold enhancement of $\varepsilon$ values is observed upon complexation of the $f a c-\operatorname{Re}(\mathrm{CO})_{3}$ core (Fig. 2). The less intense and lower energetic MLCT band in rhenium complexes is typically localized adjacent to the IL transition, usually in the range of $\sim 350-500 \mathrm{~nm} .^{29,32,33}$ To further investigate the character of the transitions involved during excitation, we performed TDDFT calculations at the PBE0/6-31G(d)/LANL2DZ level of theory. Our results indicate that a description of the broad absorption bands as mixed MLCT/IL/ILCT represents a more precise description (see the ESI $\dagger$ for computational details and electron density difference maps).

Upon excitation at $350 \mathrm{~nm}$, the complexes commonly emit in the orange range $\left(\lambda_{\max } \geq 560 \mathrm{~nm}\right)$ of the visible spectrum, giving large Stokes shifts of 210-250 nm (Fig. 2). These broad and structureless emission bands are most likely attributed to ${ }^{3}$ MLCT phosphorescence, frequently observed for rhenium tricarbonyl complexes. ${ }^{33}$ The solvent-dependent emission maxima of novel rhenium complexes shift in the order of $\lambda_{\max , 4}<$ $\lambda_{\text {max }, 6}<\lambda_{\text {max }, 5}$. Solvatochromic effects are contrary between the symmetrical complex $\mathbf{4}$ and the asymmetrical compounds $\mathbf{5}$ and 6. Whereas complex 4 exhibits a hypsochromic shift $\left(\lambda_{\max }=\right.$ $580 \mathrm{~nm} \rightarrow \lambda_{\max }=564 \mathrm{~nm}$ ) upon changing from acetonitrile to water, both asymmetric complexes emit at longer wavelengths, performing bathochromic shifts of $9 \mathrm{~nm}$ upon switching from acetonitrile to aqueous solutions (Fig. 2 and Table 1). The latter may be explained by an increase of the dipole moment upon excitation, which consequently leads to a better stabilization of formed excited triplet states in more polar solvents, ${ }^{34}$ which however is inverse in the case of $\mathbf{4}$. Another significant alteration in emission behaviour between 4 and the asymmetric complexes 5 and 6 is that 4 displays two distinct emission bands upon excitation at $350 \mathrm{~nm}$ (Fig. 2). Due to the similarity of the higher energy emission of $\mathbf{4}$ to the emission observed for ligand $\mathbf{1}$ (Fig. 2), it can be assumed that this particular luminescence band stems from excited ligand-centered states.
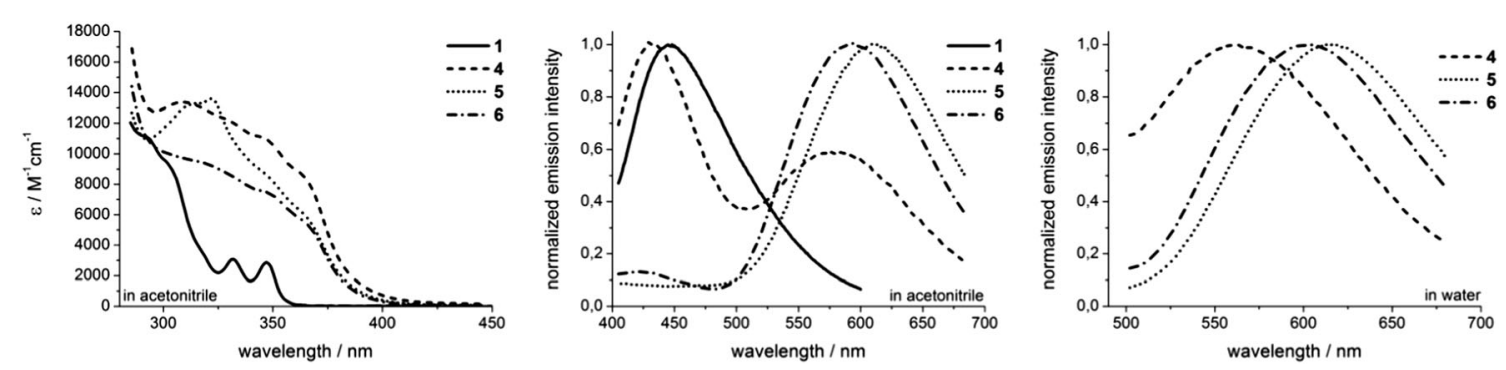

Fig. 2 Absorption spectra of ligand $\mathbf{1}$ and rhenium complexes $\mathbf{4 - 6}$ in acetonitrile (left). Normalized emission spectra in acetonitrile (middle) and in water (right). All spectra were recorded at room temperature in not degassed solvents.

Table 1 Photophysical properties of complexes 4-6 in acetonitrile and water

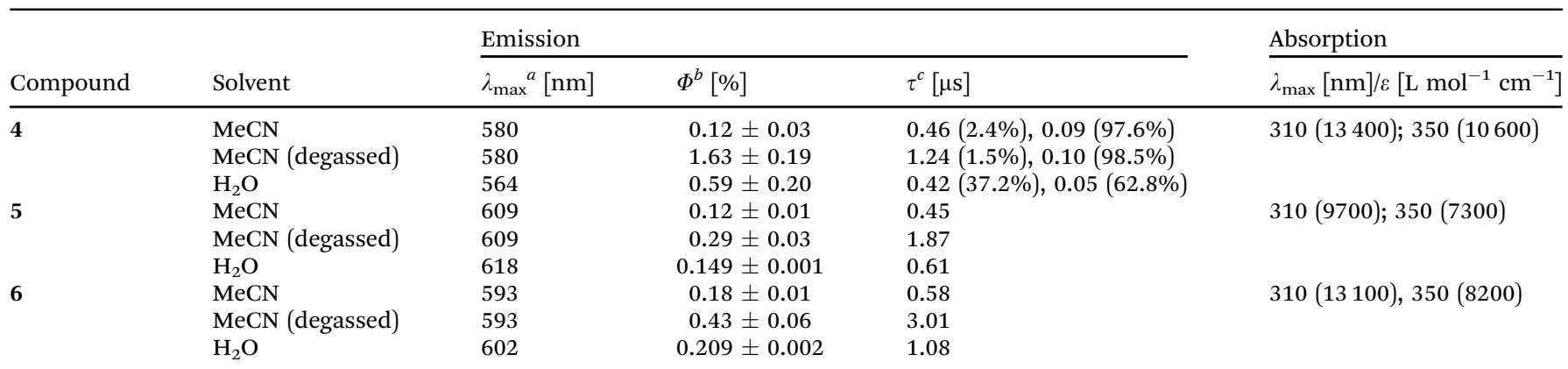

${ }^{a}$ At $298 \mathrm{~K} .^{b}$ Mean value and standard deviation from triplicates. ${ }^{c}$ Estimated errors $\pm 15 \%$. Contributions of amplitudes in bi-exponential fits are $\%$ values in brackets. 
Quantitative characterizations of the photophysical properties of complexes 4-6 show firstly typical quantum yields in non-degassed acetonitrile in the range of $0.1-0.2 \% .^{23,25,35-37}$ Simultaneously, an increase of quantum yields is observed upon switching from aerated to degassed acetonitrile. The most obvious enhancement of $\Phi$ (almost 14-fold) upon switching to degassed acetonitrile is observed for complex 4 , whereas just 2-3 fold increased quantum yields are found for the asymmetric complexes 5 and 6. When switching to aqueous solutions, the quantum yields increase by around $20 \%$ in the case of asymmetric complexes and a 5 -fold enhanced $\Phi$ value of $0.59 \%$ is found for complex 4 (Table 1). Especially for complex 4 this could be attributed to the observed ${ }^{3} \mathrm{IL}$ emission character. The influence of such a state seems to be much less pronounced in 5 and 6. Secondly, the decay in phosphorescence could be fitted to monoexponential decay curves for both asymmetric complexes $\mathbf{5}$ and $\mathbf{6}$. In contrast, the application of biexponential decay functions for correct adaptions of phosphorescence decays was necessary in the case of $\mathbf{4}$. From these curves, the emission lifetimes have been calculated (Table 1). Complex 6 displays the longest phosphorescence lifetimes, which are dependent on the solvent as well as on the presence or absence of dioxygen. In aerated acetonitrile, the ${ }^{3}$ MLCT emission lifetime is $580 \mathrm{~ns}$, which increases to $3 \mu \mathrm{s}$ in the absence of dioxygen. For complex 5, values of $450 \mathrm{~ns}$ and $1.87 \mu$ s have been obtained for aerated and degassed acetonitrile, respectively. Both asymmetric complexes are characterized by prolonged phosphorescence lifetimes in aqueous solutions with values of $1.08 \mu$ s for 6 and $609 \mathrm{~ns}$ for 5. Since the application of multiexponential decay functions is an indication for the involvement of more than just one emissive state, ${ }^{34}$ it can be assumed that complex $\mathbf{4}$ is not a pure ${ }^{3}$ MLCT emitter. Instead, it is likely that additional excited triplet states, e.g. ${ }^{3} \mathrm{IL}$, influence the emission behaviour. The obtained values reveal that short lifetime emission ( 90-100 ns) is predominant in acetonitrile solutions, whereas long-lived contributions of $460 \mathrm{~ns}$ (aerated acetonitrile) and $1.24 \mu \mathrm{s}$ (degassed acetonitrile) are mostly negligible. By switching to aqueous solutions the amount of short-lived emission (50 ns) decreases significantly, whereas the long-lived luminescence (420 ns) in turn increases. ${ }^{34}$ Overall, the luminescence lifetimes render these complexes interesting candidates for future advanced investigations in time-gated fluorescence microscopy or even fluorescence lifetime imaging (FLIM). Besides large Stokes shifts and long luminescence lifetimes, the evidence for emission from triplet states in rhenium complexes 4-6 is further underlined by the comparison of photophysical data obtained for aerated and degassed acetonitrile. Both quantum yields as well as phosphorescence lifetimes are decreased in the case of the aerated solvent, which is readily explained through quenching processes of the emissive triplet states by energy transfer to ${ }^{3} \mathrm{O}_{2} \cdot{ }^{32,38}$

With respect to the present photophysical data, ISC processes populating ${ }^{3}$ MLCT states seem to be more efficient in the case of asymmetric complexes. Whereas the excitation of the symmetric complex 4 populates fluorescence singlet states as well as mixed, radiative triplet states, 5 and $\mathbf{6}$ appear to be mostly ${ }^{3}$ MLCT emitters. The described rhenium complexes are characterized

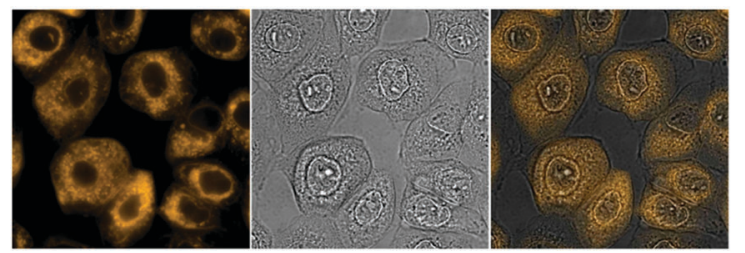

Fig. 3 Imaging experiments with $6\left(500 \mathrm{~nm}, 90 \mathrm{~min}\right.$ incubation at $37^{\circ} \mathrm{C}$, $10 \% \mathrm{CO}_{2}$ ) on IMIM-PC2 cells, left: fluorescence image (artificial colour), middle: bright field image, and right: merged image.

by solvatochromic effects, yielding improved luminescence characteristics in aqueous over acetonitrile solution. Although the solvent dependent emission maxima are contrary between 4 and asymmetric complexes, all rhenium compounds display increased quantum yields and at least the asymmetric compounds 5 and $\mathbf{6}$ demonstrate significantly prolonged emission lifetimes in aqueous solutions. The solvent dependency of emission behaviour of rhenium(I) tricarbonyl complexes has been shown to be largely influenced by the ligand structure. Although in most cases improved photophysical properties are obtained in less polar solvents, ${ }^{33}$ a few examples of opposite behaviour have been reported. ${ }^{15,39}$

With these promising photophysical data in hand, we went on to investigate the applicability of the rhenium complexes as imaging agents in live cell fluorescence microscopy. To this end, complexes 4-6 were added to IMIM-PC2 cancer cells in a concentration of $500 \mathrm{nM}$ in supplemented DMEM and after incubation at $37{ }^{\circ} \mathrm{C}$ and $10 \% \mathrm{CO}_{2}$ for $90 \mathrm{~min}$, the cells were analysed by fluorescence microscopy. The large Stokes shift enabled the application of adjusted filter sets, which in turn resulted in images of high contrast. Good staining results were obtained for all three complexes upon excitation in the ultraviolet $\left(\lambda_{\mathrm{Ex}}=326-375 \mathrm{~nm}\right)$. As a note of caution, UV excitation is generally less preferred as it is potentially damaging for cells. Luminescence which is assigned to the ${ }^{3}$ MLCT emission $\left(\lambda_{\mathrm{Em}}>510 \mathrm{~nm}\right)$ was observed, with accumulation in cytosolic, subcellular organelles (Fig. 3).

To our great delight, we observed an accumulation of the complexes around the nucleus. Colocalisation studies (complexes 4 and 6) with commercially available organelle stains confirm the enrichment within the endoplasmic reticulum (ER). In contrast to the high degree of colocalization with the ER stain (Fig. 4), colocalisation experiments with Golgi and mitochondrially localizing dyes showed significant discrepancies, thus ruling

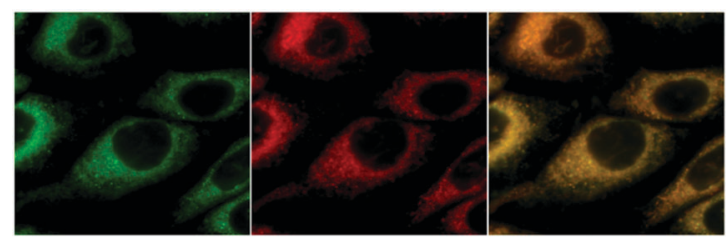

Fig. 4 Colocalisation experiments with 6 on IMIM-PC2 cells, $600 \times$ magnification. Fluorescence images are coloured artificially. Left: $\mathbf{6}$, middle: commercial ER stain ER-ID ${ }^{\mathrm{TM}}$ red, and right: merged image. 
out major colocalisation in either compartment. Since at least some selectivity of complexes for the ER has been demonstrated for IMIM-PC2 cells, we also carried out ER colocalization experiments on PT45, MCF-7, HT29, and Hep G2 cell lines. The results indicate a comparably high degree of matching regions. This confirms that selectivity for the ER is not specific for a single cell line.

Further, unlike all commercially available organelle stains, all rhenium complexes displayed no indications of photobleaching throughout the experiments (see the ESI $\dagger$ for a comparison with commercial stains). Moreover, it can be stated that the asymmetric rhenium complex 6 demonstrated the most beneficial staining and luminescence properties with shortest exposure times and brightest images.

In summary, we have shown the successful synthesis of novel phenanthridinyl based chelating ligands and their rhenium(I) tricarbonyl complexes. Moreover, we have quantified their favourable photophysical properties which show advantages over previously published compounds with respect to the emission behaviour in aqueous media. The consistent photophysical behaviour in aqueous solutions combined with the fast cellular uptake makes the complexes studied herein not only suitable as imaging agents for conventional fluorescence microscopy, but also interesting candidates for future studies employing more sophisticated lifetime-based imaging techniques. Remarkably, because of the selective accumulation in the ER, these complexes are suitable for direct ER staining.

\section{Notes and references}

1 K. K.-W. Lo, Acc. Chem. Res., 2015, 48, 2985-2995.

2 C. A. Puckett, R. J. Ernst and J. K. Barton, Dalton Trans., 2010, 39, 1159-1170.

3 F. Wang, W. B. Tan, Y. Zhang, X. Fan and M. Wang, Nanotechnology, 2006, 17, R1-R13.

4 K. K.-W. Lo, Struct. Bonding, 2007, 123, 205-245.

5 K. K.-W. Lo, K. Y. Zhang and S. P.-Y. Li, Eur. J. Inorg. Chem., 2011, 3551-3568.

6 K. L. Haas and K. J. Franz, Chem. Rev., 2009, 109, 4921-4960.

7 J.-C. G. Buenzli, Chem. Rev., 2010, 110, 2729-2755.

8 F. L. Thorp-Greenwood, R. G. Balasingham and M. P. Coogan, J. Organomet. Chem., 2012, 714, 12-21.

9 V. Fernandez-Moreira, F. L. Thorp-Greenwood and M. P. Coogan, Chem. Commun., 2010, 46, 186-202.

10 M. V. Werrett, P. J. Wright, P. V. Simpson, P. Raiteri, B. W. Skelton, S. Stagni, A. G. Buckley, P. J. Rigby and M. Massi, Dalton Trans., 2015, 44, 20636-20647.

11 M. V. Werrett, G. S. Huff, S. Muzzioli, V. Fiorini, S. Zacchini, B. W. Skelton, A. Maggiore, J. M. Malicka, M. Cocchi, K. C. Gordon, S. Stagni and M. Massi, Dalton Trans., 2015, 44, 8379-8393.

12 C. A. Bader, R. D. Brooks, Y. S. Ng, A. Sorvina, M. V. Werrett, P. J. Wright, A. G. Anwer, D. A. Brooks, S. Stagni, S. Muzzioli, M. Silberstein, B. W. Skelton, E. M. Goldys, S. E. Plush, T. Shandala and M. Massi, RSC Adv., 2014, 4, 16345-16351.
13 A. J. Amoroso, R. J. Arthur, M. P. Coogan, J. B. Court, V. FernandezMoreira, A. J. Hayes, D. Lloyd, C. Millet and S. J. A. Pope, New J. Chem., 2008, 32, 1097-1102.

14 A. J. Amoroso, M. P. Coogan, J. E. Dunne, V. Fernandez-Moreira, J. B. Hess, A. J. Hayes, D. Lloyd, C. Millet, S. J. A. Pope and C. Williams, Chem. Commun., 2007, 3066-3068.

15 M. P. Coogan, V. Fernandez-Moreira, J. B. Hess, S. J. A. Pope and C. Williams, New J. Chem., 2009, 33, 1094-1099.

16 K. K.-W. Lo, M.-W. Louie, K.-S. Sze and J. S.-Y. Lau, Inorg. Chem., 2008, 47, 602-611.

17 Y. Shen, B. P. Maliwal and J. R. Lakowicz, J. Fluoresc., 2001, 11, 315-318.

18 A. Leonidova, V. Pierroz, L. A. Adams, N. Barlow, S. Ferrari, B. Graham and G. Gasser, ACS Med. Chem. Lett., 2014, 5, 809-814.

19 C.-C. Ko, C.-O. $\mathrm{Ng}$ and S.-M. Yiu, Organometallics, 2012, 31, 7074-7084.

20 A. F. Armstrong, N. Oakley, S. Parker, P. W. Causey, J. Lemon, A. Capretta, C. Zimmerman, J. Joyal, F. Appoh, J. Zubieta, J. W. Babich, G. Singh and J. F. Valliant, Chem. Commun., 2008, 5532-5534.

21 M. Bartholoma, J. Valliant, K. P. Maresca, J. Babich and J. Zubieta, Chem. Commun., 2009, 493-512.

22 K. P. Maresca, S. M. Hillier, F. J. Femia, C. N. Zimmerman, M. K. Levadala, S. R. Banerjee, J. Hicks, C. Sundararajan, J. Valliant, J. Zubieta, W. C. Eckelman, J. L. Joyal and J. W. Babich, Bioconjugate Chem., 2009, 20, 1625-1633.

23 K. A. Stephenson, S. R. Banerjee, T. Besanger, O. O. Sogbein, M. K. Levadala, N. McFarlane, J. A. Lemon, D. R. Boreham, K. P. Maresca, J. D. Brennan, J. W. Babich, J. Zubieta and J. F. Valliant, J. Am. Chem. Soc., 2004, 126, 8598-8599.

24 N. Viola-Villegas, A. E. Rabideau, M. Bartholomä, J. Zubieta and R. P. Doyle, J. Med. Chem., 2009, 52, 5253-5261.

25 L. Wei, J. W. Babich, W. Ouellette and J. Zubieta, Inorg. Chem., 2006, 45, 3057-3066.

26 I. Kitanovic, S. Can, H. Alborzinia, A. Kitanovic, V. Pierroz, A. Leonidova, A. Pinto, B. Spingler, S. Ferrari, R. Molteni, A. Steffen, N. Metzler-Nolte, S. Wölfl and G. Gasser, Chem. - Eur. J., 2014, 20, 2496-2507.

27 G. Gasser, A. Pinto, S. Neumann, A. M. Sosniak, M. Seitz, K. Merz, R. Heumann and N. Metzler-Nolte, Dalton Trans., 2012, 41, 2304-2313.

28 L. Raszeja, A. Maghnouj, S. Hahn and N. Metzler-Nolte, ChemBioChem, 2011, 12, 371-376.

29 L. Sacksteder, A. P. Zipp, E. A. Brown, J. Streich, J. N. Demas and B. A. DeGraff, Inorg. Chem., 1990, 29, 4335-4340.

30 A. Juris, S. Campagna, I. Bidd, J. M. Lehn and R. Ziessel, Inorg. Chem., 1988, 27, 4007-4011.

31 M. Norek, J. Dresner and J. Prochorow, Acta Phys. Pol., A, 2003, 104, 425-439.

32 M. Wrighton and D. L. Morse, J. Am. Chem. Soc., 1974, 96, 998-1003.

33 R. A. Kirgan, B. P. Sullivan and D. P. Rillema, Top. Curr. Chem., 2007, 281, 45-100.

34 J. R. Lakowicz, Principles of Fluorescence Spectroscopy, Springer, 3rd edn, 2006.

35 L. Wei, J. Babich, W. C. Eckelman and J. Zubieta, Inorg. Chem., 2005, 44, 2198-2209.

36 M.-W. Louie, H.-W. Liu, M. H.-C. Lam, T.-C. Lau and K. K.-W. Lo, Organometallics, 2009, 28, 4297-4307.

37 A. O. T. Patrocinio, M. K. Brennaman, T. J. Meyer and N. Y. Murakami Iha, J. Phys. Chem. A, 2010, 114, 12129-12137.

38 A. A. Abdel-Shafi, J. L. Bourdelande and S. S. Ali, Dalton Trans., 2007, 2510-2516.

39 B. Manimaran, P. Thanasekaran, T. Rajendran, R.-J. Lin, I. J. Chang, G.-H. Lee, S.-M. Peng, S. Rajagopal and K.-L. Lu, Inorg. Chem., 2002, 41, 5323-5325. 